\title{
Acutely developing, spurious anaemia without actual blood loss. A paradigmatic case report
}

\author{
Giuseppe Lippi*1, Gianfranco Cervellin² \\ ${ }^{1}$ Section of Clinical Biochemistry, University of Verona, Verona, Italy \\ 2Emergency Department, Academic Hospital of Parma, Parma, Italy \\ *Corresponding author: giuseppe.lippi@univr.it
}

\begin{abstract}
We describe the case of a 55-year old women admitted at night to the emergency department (ED), complaining for acute abdominal pain lasting for nearly 5 hours. A first blood testing, performed immediately after admission, revealed mild anaemia. A second blood sample, drawn two hours after admission, revealed a considerable decrease of haemoglobin, haematocrit, total white blood cell and platelet counts (between 10-15\% reduction). Abdominal ultrasonography was normal and the patient had no signs or symptoms of internal or external haemorrhage. Pre-analytical and analytical errors were accurately excluded. No infusion therapy was administered. After thoughtful discussing the sequence of events, it was ascertained that the first set of blood samples was drawn with the patient in seated position, immediately after ED admission (i.e., approximately 1 min passed from standing to seated position before venipuncture), whereas the second set of blood samples was drawn with the patient lying for two hours in supine position. This case report highlights the importance of standardizing patient position before venous blood collection, along with the crucial role played by cooperation and communication between laboratory and clinical wards for identifying and troubleshooting potential causes of spurious results of in vitro diagnostic testing.
\end{abstract}

Key words: anaemia; errors; posture; preanalytical variability; case report

\section{Introduction}

The total testing process is conventionally divided into three main phases, i.e., pre-analytical, analytical and post-analytical. Several lines of evidence, accumulated over the past decades, attest that many aspects of the pre-analytical phase (i.e., those involving patient preparation, sample collection, handling and immediate management before testing) are highly vulnerable to both random and systematic errors, especially when appropriate standard operating procedures (SOPs) are not adequately followed (1). The source and the nature of many pre-analytical errors is often underestimated, under-recognized or even overlooked by both clinicians and laboratory professionals, so representing a real risk for patient safety, especially when the clinical decision making is driven by unreliable or biased results of in vitro diagnostic testing. Among the main domains of the pre-analytical phase, accurate patient preparation for testing has a vital importance for safeguarding the accuracy and repeatability of test results. The various aspects of patient preparation that should be accurately acknowledged and expectedly standardized before blood collection entail an appropriate period of fasting (i.e., lasting not less than 8-10 hours) (2), avoidance of strenuous physical activity 24-48 hours before blood drawing (3), deep knowledge about the impact of circadian variation on the measured concentration of many analytes (especially hormones) (4), as well as patient posture before and during venipuncture. This article thereby describes the paradigmatic case of spurious acutely developing anaemia due to collection of venous blood in different patient postures. 


\section{Methods}

A 55-year old women, with a medical history of irritable bowel syndrome, was admitted at 1:00 am to the emergency department (ED) of the University Hospital of Parma, complaining for acute abdominal pain lasting for nearly 5 hours. The physical exam revealed swollen abdomen and belly pain, relieving with bowel movements, but no other objective signs and symptoms.

The emergency physician ordered routine blood testing and abdominal ultrasound. Venous blood was drawn immediately for urgent clinical chemistry (Beckman Coulter AU5800; Beckman Coulter Inc., Brea, USA) and haematological testing (Sysmex XE-2100; Sysmex Inc, Kobe, Japan), and blood tubes were immediately transported to the laboratory. The blood was collected in primary blood tubes without additives for clinical chemistry measurements and containing $\mathrm{K}_{2}$ EDTA for haematological testing (both, Terumo Europe N.V., Leuven, Belgium). The main laboratory data are summarized in the Table 1.

Briefly the data showed mild anaemia (haemoglobin: $118 \mathrm{~g} / \mathrm{L}$; haematocrit: 0.35 ), normal leukocyte count (white blood cells (WBC): $3.5 \times 10^{9} / \mathrm{L}$ ), normal
WBC differential and normal values of routine clinical chemistry tests, including $C$ reactive protein. The patient was then managed conservatively, with wait-and-watch in the observation unit of the $E D$, waiting for abdominal ultrasound, and without drugs or fluid administration. A second series of venous blood samples was then drawn in the ED observation unit two hours after admission, and blood tubes were immediately transported to the laboratory. The main test results obtained in samples collected with the second venipuncture are also summarized in the Table 1.

Notably, the haemoglobin and haematocrit values both decreased by approximately $10 \%$, the total WBC and platelet counts decreased by $\sim 15 \%$, whereas the WBC differential and the other clinical chemistry parameters remained unchanged or displayed minor variations. The variation of haemoglobin, haematocrit, WBC and platelet count were all found to be clinically significant compared to the within-subject biologic variation of these parameters (i.e., $2.8 \%, 2.7 \%, 11.4 \%$ and $9.1 \%$, respectively) (5). No significant findings were found with abdominal ultrasound, and the clinical conditions of the patient remained completely stable.

TABLE 1. Laboratory testing at patient admission to the emergency department (ED) and 2 hours after admission.

\begin{tabular}{|c|c|c|c|}
\hline Parameter (unit) & ED admission & 2 hours after ED admission & Local reference range \\
\hline Haemoglobin (g/L) & 118 & 107 & $120-160$ \\
\hline Haematocrit (L/L) & 0.35 & 0.31 & $0.36-0.47$ \\
\hline White blood cells (x 109/L) & 3.5 & 3.0 & $4.0-11.0$ \\
\hline Neutrophils (\%) & 48 & 49 & $41-74$ \\
\hline Lymphocytes (\%) & 41 & 41 & $18-48$ \\
\hline Monocytes (\%) & 6 & 5 & $4-15$ \\
\hline Eosinophils (\%) & 3 & 3 & $1-8$ \\
\hline Basophils (\%) & 2 & 2 & $0.1-2.0$ \\
\hline Platelets (x 109/L) & 252 & 217 & $150-400$ \\
\hline $\mathrm{C}$ reactive protein $(\mathrm{mg} / \mathrm{L})$ & 1.2 & 1.0 & $<3.0 \mathrm{mg} / \mathrm{L}$ \\
\hline Creatinine $(\mu \mathrm{mol} / \mathrm{L})$ & 76 & 72 & $62-115$ \\
\hline Glucose (mmol/L) & 5.1 & 5.0 & $3.9-5.8$ \\
\hline Potassium (mmol/L) & 4.2 & 4.2 & $3.5-5.0$ \\
\hline Sodium (mmol/L) & 141 & 141 & $136-145$ \\
\hline Chloride (mmol/L) & 104 & 104 & $98-106$ \\
\hline
\end{tabular}




\section{Interventions and further investigations}

In the lack of signs and symptoms of internal or external haemorrhage, the emergency physicians called the laboratory, to discuss the possible reasons underlying the acutely developing anaemia or for troubleshooting potential preanalytical and analytical errors. Repeated analyses on the first set of blood samples gave identical results as those generated upon ED admission, so that an analytical error could be ruled out. Once fluid contamination from infusion routes, identification errors, presence of fibrin or clots in the EDTA sample were also clearly excluded, a third series of venous blood samples was collected and the analysis of these specimens generated results virtually identical to those of the second set of blood samples (data not shown).

\section{What happened?}

It was only after thoughtful discussing the sequence of events from patient admission to performance of the second venipuncture that both the emergency physician and the laboratory professional discovered that the first series of blood samples had been drawn with the patient in seated position, immediately after arrival in the ED (i.e., approximately $1 \mathrm{~min}$ passed from standing to seated position for venipuncture), whereas the second set of blood samples had been drawn with the patient lying on a gurney, in supine posture, for nearly two hours. This actually explained the substantial variation of haemoglobin, haematocrit and WBC count, since the time passed from upright to seated position was not sufficient to stabilize the compartmental distribution of body fluids. The patient was finally diagnosed with exacerbation of irritable bowel syndrome and discharged with a prescription for analgesic drugs.

\section{Discussion}

Despite the impact of many pre-analytical variables on quality of laboratory testing has been thoughtfully described over the past decades, this part of the total testing process remains highly error-prone. The phlebotomy practice is especially vulnerable to errors and other non-conformities (6), and this is mostly attributable to the fact that the quality of current practices is low, phlebotomists' training may be insufficient or inadequate, or else that compliance with available guidelines for drawing blood is poor $(7,8)$.

The potential impact of patient posture before and during venipuncture has been recognized for decades. In 1966, Stoker et at., first showed that 15 min of standing in upright position caused a substantial increase (up to 13\%) of total cholesterol in plasma, accompanied with comparable increases of total proteins and haematocrit (9). In 1977, Humphrey et al. also demonstrated that the values of calcium and total protein in serum were $2 \%$ to $7 \%$ lower when blood was collected in recumbent posture compared to upright position (10). The following year, Dixon and Paterson also published an interesting study showing that plasma proteins, calcium, bilirubin, alkaline phosphatase, cholesterol and other molecules bound to the proteins may be substantially decreased (up to - 17\%) when blood is collected in supine compared to standing posture (11). Some other ensuing studies were then published in the 1970-1980s, substantially confirming this data. More recently, Lippi et al. performed another study using modern blood collection devices and contemporary laboratory instrumentation, showing that the results of a number of laboratory tests display both analytically and clinically significant decreases when the venipuncture is performed in supine compared to standing position (12). In particular, haemoglobin and haematocrit were shown to exhibit rather ample variations after shifting from one position to another, approximating $10 \%$ decrease in blood samples collected in recumbent posture compared to upright position. This is mainly due to the fact that gravitational force has a strong impact on venous hydrostatic changes and compartmental distribution of body fluids. Prolonged stay in upright position causes plasma extravasation, diffusion of filterable elements and plasma in the interstitial space, whereas bigger and substantially non-diffusible components of serum or plasma remain within the blood vessels, so leading to an increased concentration (13). This provides a reason- 
able explanation to the data observed in our case report, with blood cells and large molecules (i.e., haemoglobin, $C$ reactive protein and creatinine) displaying decreased values and filterable elements (i.e., ions) remaining virtually unchanged from upright to supine patient posture.

When these findings are translated into a realworld scenario, such as that described in our case report, they may generate a substantial impact on patient management and safety. An episode of acute blood loss, regardless of the underlying cause and especially when occurring in surgical patients, may in fact trigger a number of inappropriate and potentially invasive investigations (i.e., $\mathrm{X}$-rays, gastroscopy, colonoscopy, computed tomography, laparoscopy), or may induce inappropriate patient management (e.g., blood transfusions) (14), especially when pre-analytical and analytical problems are not recognized.

Despite the many national and international guidelines on collection of diagnostic blood specimens substantially agree about the fact that patient posture should be standardized before and during venipuncture (15-18), their effective implementation throughout different healthcare settings, as well as the compliance with these, are both dramatically insufficient (19). This is especially true in the ED, where overcrowding and frenetic activities may lead the personnel to disregard SOPs for blood collection and overlook the best practices $(1,20)$.
The leading take-home messages of this case report are that: (i) indications of official guidelines and recommendations for drawing venous blood should be implemented and more stringently applied throughout all the different healthcare settings, including the ED; (ii) blood collection should be always performed with the patient in the same position; (iii) the patient posture should be stabilized to one (i.e., sitting) or another (i.e., supine) position for at least 10-15 min before drawing blood; (iv) when receiving test results that do not actually match with the patient conditions, the possible impact of one or more preanalytical variables (or analytical errors) should be thoughtfully excluded; (v) cooperation and communication between the laboratory and the clinical wards (so including the ED) is the mainstay for identifying and troubleshooting the cause of spurious results of in vitro diagnostic testing. Unfortunately, we acknowledge that the ideal procedure for collecting blood cannot be always followed, especially in short stay units such as the ED, and so the potential clinical impact of using poorly standardized practices may be clearly acknowledged. Therefore, whenever clinically unjustified variations of laboratory parameters occur, the clinicians should promptly contact the laboratory staff to troubleshoot potential pre-analytical causes. It seems also reasonable to suggest that the patient posture may be checked and recorded along with full demographic data during blood drawing.

\section{Potential conflict of interest}

None declared.

\section{References}

1. Simundic AM, Lippi G. Preanalytical phase - a continuous challenge for laboratory professionals. Biochem Med ( $\mathrm{Za}-$ greb) 2012;22:145-9. https://doi.org/10.11613/BM.2012.017

2. Simundic AM, Cornes M, Grankvist K, Lippi G, Nybo M. Standardization of collection requirements for fasting samples: for the Working Group on Preanalytical Phase (WG-PA) of the European Federation of Clinical Chemistry and Laboratory Medicine (EFLM). Clin Chim Acta 2014;432:33-7. https:// doi.org/10.1016/j.cca.2013.11.008

3. Sanchis-Gomar F, Lippi G. Physical activity - an important preanalytical variable. Biochem Med (Zagreb) 2014;24:6879. https://doi.org/10.11613/BM.2014.009

4. van Kerkhof LW, Van Dycke KC, Jansen EH, Beekhof PK, van Oostrom CT, Ruskovska T, et al. Diurnal Variation of Hormonal and Lipid Biomarkers in a Molecular Epidemiology-Like Setting. PLoS One 2015;10:e0135652. https://doi. org/10.1371/journal.pone.0135652 
5. Ricós C, Alvarez V, Cava F, García-Lario JV, Hernández A, Jiménez $C V$, et al. Current databases on biological variation: pros, cons and progress. Scand J Clin Lab Invest 1999;59:491500. https://doi.org/10.1080/00365519950185229

6. Ialongo C, Bernardini S. Phlebotomy, a bridge between laboratory and patient. Biochem Med (Zagreb) 2016;26:1733. $h$ ttps://doi.org/10.11613/BM.2016.002

7. Simundic AM, Cornes M, Grankvist K, Lippi G, Nybo M, Kovalevskaya $S$, et al. Survey of national guidelines, education and training on phlebotomy in 28 European countries: an original report by the European Federation of Clinical Chemistry and Laboratory Medicine (EFLM) working group for the preanalytical phase (WG-PA). Clin Chem Lab Med 2013;51:1585-93. https://doi.org/10.1515/cclm-2013-0283

8. Simundic AM, Church S, Cornes MP, Grankvist K, Lippi G, Nybo $M$, et al. Compliance of blood sampling procedures with the CLSI H3-A6 guidelines: An observational study by the European Federation of Clinical Chemistry and Laboratory Medicine (EFLM) working group for the preanalytical phase (WG-PRE). Clin Chem Lab Med 2015;53:1321-31. https://doi.org/10.1515/cclm-2014-1053

9. Stoker DJ, Wynn V, Robertson G. Effect of posture on the plasma cholesterol level. Br Med J 1966;1:336-8. https://doi. org/10.1136/bmj.1.5483.336

10 .Humphrey KR, Gruemer HD, Lott JA. Impact of posture on the "reference range" for serum proteins and calcium. Clin Chem 1977;23:1343-6.

11. Dixon $M$, Paterson CR. Posture and the composition of plasma. Clin Chem 1978;24:824-6.

12. Lippi G, Salvagno GL, Lima-Oliveira G, Brocco G, Danese $E$, Guidi GC. Postural change during venous blood collection is a major source of bias in clinical chemistry testing. Clin Chim Acta 2015;440:164-8. https://doi.org/10.1016/j. cca.2014.11.024
13. Hagan RD, Diaz FJ, Horvath SM. Plasma volume changes with movement to supine and standing positions. J Appl Physiol Respir Environ Exerc Physiol 1978;45:414-7.

14. Kahsai D. Acute Anemia. Available at: http://emedicine. medscape.com/article/780334-overview. Accessed: February 22 nd 2017.

15. Lippi G, Caputo M, Banfi G, Buttarello M, Ceriotti F, Daves M, et al. Recommendations for collection of venous blood. RIMeL - IJLAM 2008;4:249-58.

16. Clinical Laboratory Standards Institute. Procedures for collection of diagnostic blood specimens by venipuncture approved guideline - 6th ed. CLSI document H3-A6. Wayne, PA: Clinical and Laboratory Standards Institute; 2007.

17. World Health Organization. WHO guidelines on drawing blood: best practices in phlebotomy. WHO Press, World Health Organization, Geneva, Switzerland; 2010.

18. Nikolac N, Supak-Smolčić V, Šimundić AM, Ćlap I; Croatian Society of Medical Biochemistry and Laboratory Medicine. Croatian Society of Medical Biochemistry and Laboratory Medicine: national recommendations for venous blood sampling. Biochem Med (Zagreb) 2013;23:242-54. https:// doi.org/10.11613/BM.2013.031

19. Nikolac N, Simundic AM, Kackov S, Serdar T, Dorotic A, Fumic $K$, et al. The quality and scope of information provided by medical laboratories to patients before laboratory testing: Survey of the Working Group for Patient Preparation of the Croatian Society of Medical Biochemistry and Laboratory Medicine. Clin Chim Acta 2015;450:104-9. https:// doi.org/10.1016/j.cca.2015.08.001

20. Lippi G, Banfi G, Church S, Cornes M, De Carli G, Grankvist K, et al. In pursuit of harmony, on behalf of European Federation for Clinical Chemistry and Laboratory Medicine (EFLM) Working group for Preanalytical Phase (WG-PRE). Clin Chem Lab Med 2015;53:357-70. https://doi.org/10.1515/ cclm-2014-1051 